\title{
Exactly massless fermions on the Lattice
}

\author{
Ting-Wai Chiu ${ }^{\text {* * }}$
}

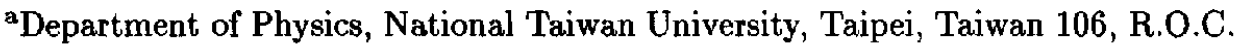

The salient features of the Ginsparg-Wilson fermion in topologically nontrivial background gauge fields are outlined. The $R$-invariance of the zero modes, the indices and the index theorem on a finite lattice are illustrated. The role of $R$ in converting the nonlocal $D_{c}$ into a sequence of highly local $D$ is demonstrated.

\section{INTRODUCTION}

My talk at Lattice 98 essentially consisted of two parts : (1) The general solution of the Ginsparg-Wilson (GW) relation; (2) Some numerical results for the (generalized) NeubergerDirac fermion operator. In this writeup, due to the page limit, I confine my discussions to the second part, which I did not have enough time to cover in my talk. For the first part, I refer to ref. [5] and ref. [6] for details. Although the numerical results are obtained for the (generalized ) Neuberger-Dirac fermion operator which is so far the only explicit and physical solution of the $G W$ relation, nevertheless, they can serve the purpose to illustrate the general properties of the GW fermion.

The salient feature of the GW fermion $D$

$D \gamma_{5}+\gamma_{5} D=2 D R \gamma_{5} D$

is that its chiral symmetry breaking part [ the RHS of (1) ] does not change anything of the zero modes of $D$ for any $R$ which controls the amount of chiral symmetry breaking as well as the locality of $D$. Therefore any zero mode of $D$ must be a zero mode of $D_{c}$, the chiral symmetric fermion operator in the chiral limit $R \rightarrow 0$, and vice versa. This can be easily seen by considering the general solution $[5,6]$ of Eq. (1)

$D=D_{c}\left(\mathbf{I}+R D_{c}\right)^{-1}=\left(\mathbf{I}+D_{c} R\right)^{-1} D_{c}$

where

$D_{c} \gamma_{5}+\gamma_{5} D_{c}=0$

*This work is supported by the National Science Council, R.O.C. under the grant numbers NSC87-2112-M002-013 and NSC88-2112-M002-016
Consequently, $\quad N_{+}[D, A]=N_{+}\left[D_{c}, A\right]$ and $N_{-}[D, A]=N_{-}\left[D_{c}, A\right]$ where $N_{+}\left[D_{\mathrm{c}}, A\right]$ ( $\left.N_{-}\left[D_{c}, A\right]\right)$ is the number of zero modes of positive ( negative ) chirality, a functional of $A$ and $D_{c}$. The index of $D$ is equal to the index of $D_{c}$, $\operatorname{ind}(D) \equiv N_{-}[D, A]-N_{+}[D, A]=\operatorname{ind}\left(D_{c}\right)$. These results hold for any $R$. According to the NielsonNinomiya no-go theorem [2], in the chiral limit $R \rightarrow 0$, so if we want to keep $D_{c}$ free of species doubling, then $D_{c}$ must be non-local. The nonlocality is nothing to be afraid of, and in fact we can live with it, and tame it to be harmless and even useful. We will show that the zero modes of $D_{c}$ agree with the continuum solution excellently and the index theorem is realized exactly on a finite lattice. Then we will turn on $R$ and show that all topological characteristics of $D_{c}$ remain invariant while the nonlocal $D_{c}$ is transformed into a sequence of local $D^{\prime} s$. It has been shown in ref. [6] that any well defined $D_{c}$ must have zero index. In order to have nonzero indices, $D_{c}$ must be singular (i.e., divergent) in topologically nontrivial background gauge field. Although all these properties have been discussed analytically in ref. [6], nevertheless, it is instructive to convey this picture vividly through some numerical examples in this paper. More details of these studies will be reported later [7].

\section{GENERALIZED NEUBERGER OPERATOR.}

Consider the Neuberger-Dirac operator [3] in the chiral limit $(R \rightarrow 0)$

$D_{c}=2 M \frac{\mathbf{I}+V}{\mathbf{I}-V}, \quad V=D_{w}\left(D_{w}^{\dagger} D_{w}\right)^{-1 / 2}$ 
where $D_{w}$ is the Wilson-Dirac fermion operator with negative mass $-M$ and Wilson parameter $r_{w}>0$

$D_{w}=-M+\frac{1}{2}\left[\gamma_{\mu}\left(\nabla_{\mu}^{*}+\nabla_{\mu}\right)-r_{w} \nabla_{\mu}^{*} \nabla_{\mu}\right]$

where $\nabla_{\mu}$ and $\nabla_{\mu}^{*}$ are the forward and backward difference operators. Using Eq. (2), we obtain the generalized Neuberger-Dirac operator

$D=2 M(\mathbf{I}+V)[(\mathbf{I}-V)+2 M R(\mathbf{I}+V)]^{-1}$

For $M \in\left(0,2 r_{w}\right), D$ is topologically non-trivial. In the following, we restrict our discussions to the simplest $R$ which is proportional to the identity in the position space and trivial in the Dirac space. It was first demonstrated in ref. [8] that for $R=1 / 2$ and in two dimensional $U(1)$ background gauge fields, $D$ reproduces exact zero modes with definite chirality on a finite lattice, the index theorem is satisfied exactly, and the fermionic determinants are also in good agreement with the continuum exact solutions. In this paper, we again fix $M=1$ and $r_{w}=1$, and explicitly verify that the Atiyah-Singer index theorem

$Q=N_{-}[D, A]-N_{+}[D, A]$

holds exactly for all values of $R$, say, from $10^{-6}$ to $10^{6}$, in topologically nontrivial $U(1)$ and $S U(2)$ background gauge fields, on two dimensional and four dimensional lattices respectively. The two dimensional $U(1)$ background gauge field has been discussed in ref. [8] and here we use the same notations as defined in Eqs. (7)-(11) in ref. [8]. We refer to ref. [7] for details of four dimensional results.

\section{NUMERICAL EXAMPLES}

In Fig. 1, the exact zero mode with negative chirality for $D$ in topological charge $Q=1$ background gauge field on a $12 \times 12$ lattice with periodic boundary conditions is plotted. The agreement with the continuum exact solution [4] is excellent for any value of $R$ ranging from $10^{-6}$ to $10^{6}$. This verifies that the zero modes of any Ginsparg-Wilson fermion $D$ are $R$-invariant. It is striking to see that the zero mode of $D$ at $R=10^{-6}$, so close to the chiral limit, is still in excellent agreement with the continuum exact solution. In fact, the nonlocality of $D_{c}$ does not prevent it from having exact zero modes and satisfying the index theorem. Since at the chiral limit, the Ginsparg-Wilson relation is turned off, the existence of such a topologically nontrivial $D_{c}$ of course cannot be answered by the GW relation. However, if we have already found such a $D_{c}$, then we can tame its nonlocality and singularity using Eq. (2) by choosing a sufficiently large $R$. In Fig. 3, the absolute value of matrix elements, $|D(x, y)|$, versus $|x-y|$ are plotted for a topologically nontrivial gauge configuration on a $8 \times 8$ lattice with antiperiodic boundary conditions. At $R=10^{-6}, D$ is nonlocal and some elements become very large. Then at $R=0.1, D$ has been tamed a lot but it is still far from local. For $R>0.5, D$ has become local and can be fitted by an exponential decay function. If we increase $R$ further, say, $R=10.0$, then $D$ becomes almost ultralocal, i.e., $D(x, x+\hat{\mu}) \sim 10^{-3} \times D(x, x)$, $D(x, x+\hat{\mu}+\hat{\nu}) \sim 10^{-3} \times D(x, x)$, and other matrix elements $\sim 10^{-6} \times D(x, x)$. After $D$ becomes local, the fermion propagator remains almost the same for any larger $R^{\prime} s$. In Fig. 2, we plot the ( subtracted ) fermion propagator $D^{-1}(x, y)=\gamma_{\mu} S_{\mu}(x, y)$ ( cf. Eq. (20) of ref. [8] ) for $R=10.0$ on a $8 \times 8$ lattice, in a topologically trivial background gauge field with $h_{1}=0.1$ and $h_{2}=0.2$. The agreement with the continuum exact solution ( solid lines ) is excellent.

\section{SUMMARY and CONCLUSIONS}

To summarize, we have demonstrated that the nonlocality of a chirally symmetric Dirac operator $D_{c}$ on the lattice is one of the necessary requirements for it to be free of species doubling and topologically nontrivial, i.e., to have exact zero modes and to realize the Atiyah-Singer index theorem on the lattice. The best way to tame the nonlocality of $D_{c}$ is to break the chiral symmetry via the Ginsparg-Wilson solution Eq. (2) which gaurantees that the topological characteristics of $D_{c}$ is invariant for any $R$. In principle, we can make $D$ almost ultralocal by simply increasing $R$. Once $D$ becomes local enough, the fermion propagator $D^{-1}(x, y)$ remains almost the same 


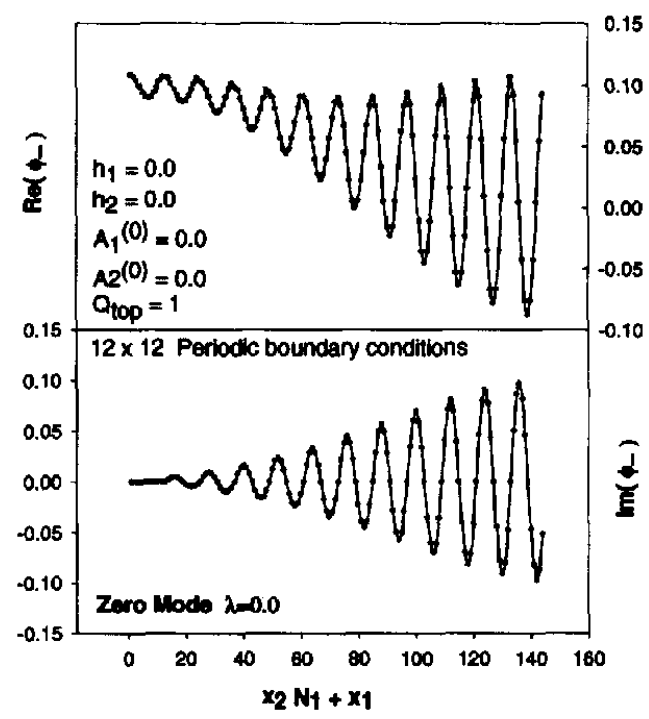

Figure 1. The real part and imaginary part of the zero mode of $D$ for $R=10^{-6}$. They remain the same for all values of $R$ from $10^{-6}$ to $10^{6}$. The solid lines denote the continuum exact solution.

for all larger $R^{\prime} s$ except the diagonal elements $D_{\alpha \alpha}^{-1}(x, x)=R$, which can be easily subtracted off. The $R$-invariance of the zero modes and the Atiyah-Singer index theorem has been verified for any value of $R$ ranging from $10^{-6}$ to $10^{6}$.

\section{REFERENCES}

1. P. Ginsparg and K. Wilson, Phys. Rev. D25 (1982) 2649.

2. H.B. Nielsen and N. Ninomiya, Nucl. Phys. B185 (1981) 20; B193 (1981) 173.

3. H. Neuberger, Phys. Lett. B417 (1998) 141; Phys. Lett. B 427 (1998) 353.

4. I. Sachs and A. Wipf, Helv. Phys. Acta 65 (1992) 652.

5. T.W. Chiu and S.V. Zenkin, hep-lat/9806019.

6. T.W. Chiu, hep-lat/9809013.

7. T.W. Chiu, NTUTH-98-108, in preparation.

8. T.W. Chiu, Phys. Rev. D58, 074511 (1998).

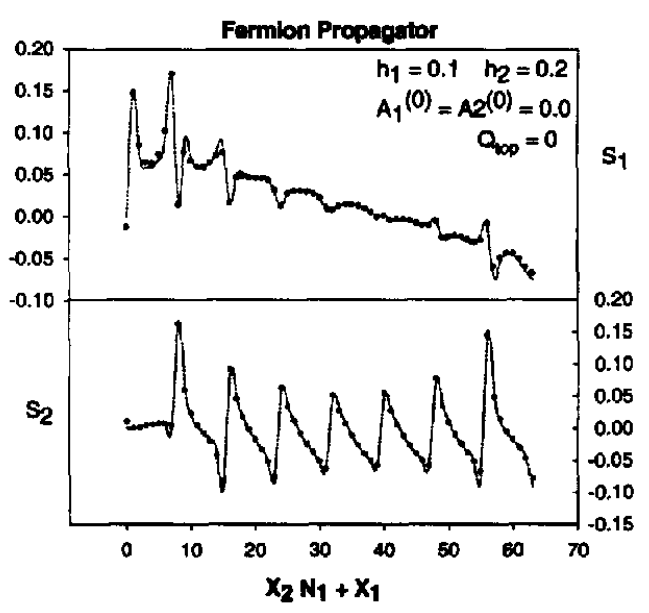

Figure 2. The fermion propagator $D^{-1}(x, 0)=$ $\gamma_{\mu} S_{\mu}(x, 0)$ for $R=10.0$ on a $8 \times 8$ lattice with antiperiodic boundary conditions.

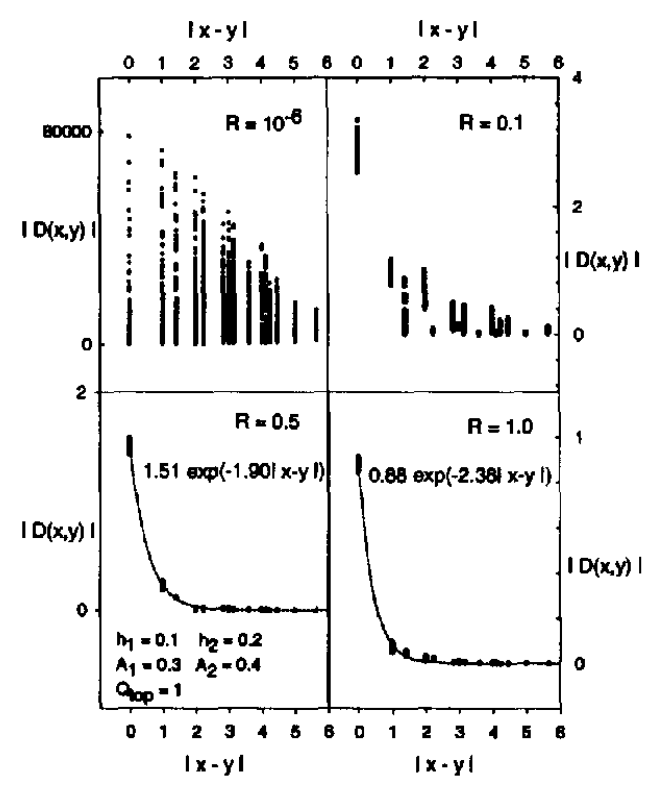

Figure 3. The locality of $D$ versus $R$ in a topologically nontrivial background gauge field. 